\title{
Cetoacidose diabética em crianças: revisão da fisiopatologia e tratamento com o uso do "método de duas soluções salinas"
}

\author{
Diabetic ketoacidosis in children: review of pathophysiology \\ and treatment with the use of the "two bags system"
}

Paulo Ferrez Collett-Solberg*

\section{Resumo}

Objetivo: Rever vários aspectos da cetoacidose diabética, incluindo o "sistema de duas soluções", um método de administrar líquidos que proporciona correção mais suave do estado de hiperglicemia e de cetose.

Métodos: Revisão de publicações recentes (dos últimos 7 anos) referidas na base de dados da Medline e capítulos de livros de pediatria que abordam a etiologia, o tratamento e as complicações da cetoacidose diabética. $\mathrm{O}$ tratamento aqui apresentado utiliza a informação encontrada nestes artigos e a experiência clínica adquirida no Children's Hospital of Philadelphia e no Duke University Medical Center.

Resultados: A patologia do diabetes mellitus tipo 1 envolve a destruição das células $\beta$ do pâncreas, causando uma deficiência de insulina. A insulina é essencial para o metabolismo de carboidratos, proteínas e gorduras. A deficiência de insulina pode levar a um quadro de cetoacidose diabética que apresenta três componentes: 1) a hiperglicemia, que causa glicosúria e conseqüentemente desidratação; 2) a lipólise em excesso, que causa aumento nos níveis séricos e urinários de corpos cetônicos; e 3) a acidose metabólica, que é causada pela desidratação e pela presença de corpos cetônicos no sangue. A cetoacidose diabética é uma condição grave que, se não tratada adequadamente, pode levar ao coma e à morte. Em crianças, uma das mais temidas complicações é o edema cerebral.

Conclusões: Na literatura publicada existem diversas maneiras de se tratar a cetoacidose diabética em pediatria, sem um consenso sobre a causa da principal complicação, edema cerebral, e conseqüentemente, sem um consenso quanto ao melhor método de intervenção. $\mathrm{O}$ uso do sistema de duas soluções em pacientes em cetoacidose diabética permite o rápido ajuste na concentração da glicose infundida, simplificando e barateando o tratamento da cetoacidose diabética.

J Pediatr (Rio J) 2001; 77(1): 9-16: diabetes mellitus, cetoacidose diabética, edema cerebral, reposição hídrica.

\begin{abstract}
Objectives: To review diabetic ketoacidosis, including the "two bags system", a method of administering liquids in order to provide a smoother correction of the hyperglycemic and ketotic states.

Methods: Review of recent publications (last 7 years) from a Medline search and chapters published in pediatric textbooks that discuss the etiology, therapy, and complications of diabetic ketoacidosis. The management approach incorporates the findings of these publications as well as the clinical experience at the Children's Hospital of Philadelphia and Duke University Medical Center.

Results: The pathology of the type 1 Diabetes Mellitus involves the progressive destruction of the $\beta$ cells of the pancreas, causing insulin deficiency. Insulin is essential in the metabolism of carbohydrates, protein and fat. Insulin deficiency may lead to diabetic ketoacidosis which has three components: 1) hyperglycemia, which causes glycosuria and consequently dehydration; 2) lipolysis which, causes ketonemia/ketonuria; and 3) acidosis, that is caused by the dehydration and the high serum levels of ketones. Diabetic ketoacidosis is a serious condition and, if not treated appropriately, can cause coma and death. In children cerebral edema is the major complication of the therapy for diabetic ketoacidosis. Careful replacement of insulin, fluids, glucose and electrolytes is essential.
\end{abstract}

Conclusions: The literature presents different ways to manage DKA in pediatrics, without a consensus on the cause of the most important complication (cerebral edema), and consequently without a consensus on the best approach.

The use of the two saline bags in patients in DKA allows fast adjustments in the dextrose concentration of the infusion fluids, simplifying and reducing the costs of the treatment of diabetic ketoacidosis.

J Pediatr (Rio J) 2001; 77(1): 9-16: diabetes mellitus, cerebral edema, diabetic ketoacidosis, fluid therapy.

\footnotetext{
* Professor Assistente, Divisão de Endocrinologia, Depto. de Pediatria - Duke University Medical Center, EUA. Este trabalho teve o apoio da Genentech Foundation for growth and development.
} 


\section{Introdução}

O diabetes mellitus tipo 1 é uma doença auto-imune que afeta milhares de crianças pelo mundo. A prevalência é de 1 em 2500 crianças com menos de 5 anos de idade e de $1 \mathrm{em}$ 300 pessoas aos 18 anos. Há uma variação regional/racial importante, tendo uma incidência de $30-40$ casos por 100.000 crianças na Finlândia e de menos de 1 por 100.000 crianças entre os orientais. Estudos feitos em São Paulo mostraram uma incidência de 3,6 por 100.000 .

A patologia do diabetes mellitus tipo 1 envolve a destruição progressiva das células $\beta$ do pâncreas, levando a uma deficiência de insulina e a alterações daí resultantes. $\mathrm{O}$ quadro de hiperglicemia associada a um aumento na produção de corpos cetônicos é conhecido como cetoacidose diabética (CAD).

A hiperglicemia leva à glicosúria e à desidratação, enquanto que a produção excessiva de corpos cetônicos agrava a acidose metabólica decorrente da desidratação. A cetoacidose diabética, se não tratada adequadamente, pode levar ao coma e à morte. Em crianças, a principal complicação da terapia para a cetoacidose diabética é o edema cerebral.

Nesta revisão discutiremos vários aspectos da cetoacidose diabética, incluindo uma maneira diferente de administrar líquidos, a fim de proporcionar uma correção mais suave do estado de hiperglicemia e cetose. $\mathrm{O}$ intuito deste trabalho não é proporcionar uma "receita de bolo" a ser seguida quando frente a frente com um paciente com CAD, já que a variação nas formas de apresentação e na resposta à terapia é muito grande, e diferentes centros e especialidades apresentam protocolos diversos. O nosso objetivo é fazer uma revisão geral neste importante tópico e ao mesmo tempo apresentar um enfoque sobre o "sistema de duas soluções", que simplifica, agiliza e barateia o tratamento de CAD.

\section{Definição}

Não existe um consenso na literatura sobre a definição de cetoacidose diabética. A cetoacidose diabética está presente quando existe uma acidose metabólica com um pH arterial abaixo de 7,3 ou um bicarbonato plasmático abaixo de $15 \mathrm{mEq} / \mathrm{dl}$, e um aumento inapropriado na concentração de corpos cetônicos no sangue e, conseqüentemente, na urina. A glicemia normalmente está elevada, acima de 240 $\mathrm{mg} / \mathrm{dl}$, mas pode estar normal.

\section{Incidência}

Vinte e cinco porcento das crianças, quando diagnosticadas com diabetes mellitus, estão em cetoacidose, sendo que $15 \%$ destas crianças apresentam um quadro grave. Em pacientes sabidamente diabéticos, incluindo adultos e crianças, a cetoacidose diabética ocorre em 0,2 a $8,0 \%$ dos pacientes por ano. A mortalidade é mais elevada em adultos devido à presença de anormalidades em outros órgãos, ocorrendo em 2 a $5 \%$ dos episódios em adultos e em 1 a 3\% dos episódios em crianças 5 .

\section{Fatores predisponentes}

A cetoacidose diabética é um quadro progressivo e conseqüentemente, quanto mais prolongado e severo for o período de insulinopenia, maior a gravidade do quadro de CAD.

Em crianças sem um diagnóstico prévio de diabetes mellitus, o espaço de tempo entre o início dos sintomas e a intervenção médica é o principal fator que determinará a gravidade do quadro no momento do diagnóstico. Como o processo de destruição das células $\beta$ é um processo lento, normalmente as crianças apresentam um quadro de perda de peso, hiperfagia, polidipsia e poliúria por algumas semanas, às vezes meses. A capacidade da família em perceber essas alterações comportamentais da criança e a capacidade do pediatra em fazer rapidamente o diagnóstico correto são fundamentais ${ }^{7}$.

Em crianças já com um diagnóstico de diabetes mellitus, a falta do uso adequado da insulina é a causa mais comum de cetoacidose diabética. A falta de insulina pode ser real ou relativa ${ }^{7}$. A falta real é quando a criança ou os pais não seguem a recomendação médica, não administrando as doses diárias e rotineiras de insulina. A falta relativa ocorre em condições em que a necessidade de insulina aumenta, e o paciente não corrige a dose apropriadamente. Por exemplo, quadros infecciosos estão associados a um aumento na resistência à insulina. Nestas situações, deve-se sempre recomendar um monitoramento mais intenso da glicemia e da presença de corpos cetônicos na urina. Se o paciente não monitora a presença desses corpos cetônicos na urina durante esses quadros infecciosos, a perda de apetite pode mascarar o início de uma cetoacidose diabética por não causar uma elevação na glicemia.

Ensinar o paciente e a família como proceder frente a infecções e alterações na ingestão de carbohidratos é parte fundamental do trabalho do endocrinologista no controle de diabéticos. De maneira geral, a CAD é sempre decorrente da falta de insulina, pois as variações na dieta não estão associadas à produção de corpos cetônicos, a não ser quando associadas a uma diminuição da dose diária de insulina.

\section{Fisiopatologia}

As principais causas para as alterações metabólicas encontradas na cetoacidose diabética são: 1) a perda do transporte de glicose para dentro dos tecidos periféricos, como músculos e gordura, já que este transporte depende de insulina, 2) aumento da gluconeogênese e glicogenólise hepáticas, e 3) disinibição da quebra de gordura, proteínas e glicogênio (Figura 1). Com isso, a deficiência de insulina leva a uma hiperglicemia (devido a uma diminuição da 


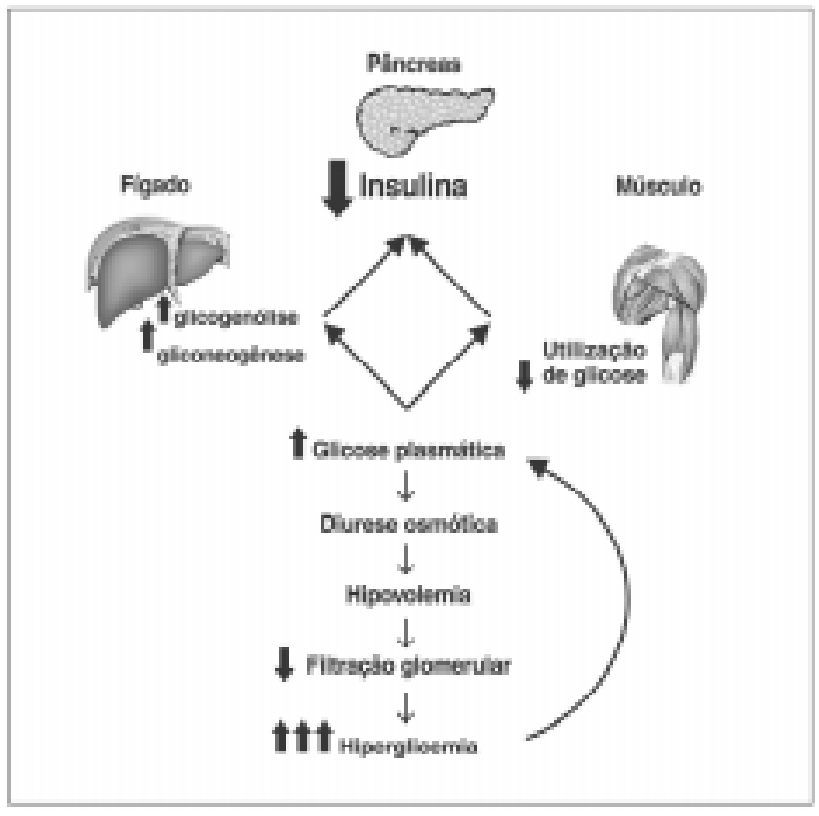

Figura 1 - Efeitos da deficiência de insulina

utilização periférica e ao aumento da produção hepática de glicose) e à acidose devido à produção de corpos cetônicos pelo fígado. Agravando este quadro, a hiperglicemia causa glicosúria e conseqüente poliúria. Essa perda hídrica é acompanhada de uma perda de eletrólitos e desidratação. Na presença de uma desidratação importante, ocorre uma diminuição da circulação periférica e aumento da produção de ácido láctico, piorando a acidose metabólica já presente (Figura 2)

Outros hormônios também estão envolvidos na CAD (Figura 3). A falta de insulina induz à liberação pancreática de glucagon. O stress e a diminuição da oferta de glicose para o meio intracelular estimulam a liberação de hormônio de crescimento, cortisol e catecolaminas. Esses hormônios vão causar um aumento da resistência aos efeitos da insulina, diminuindo ainda mais a utilização de glicose pelos

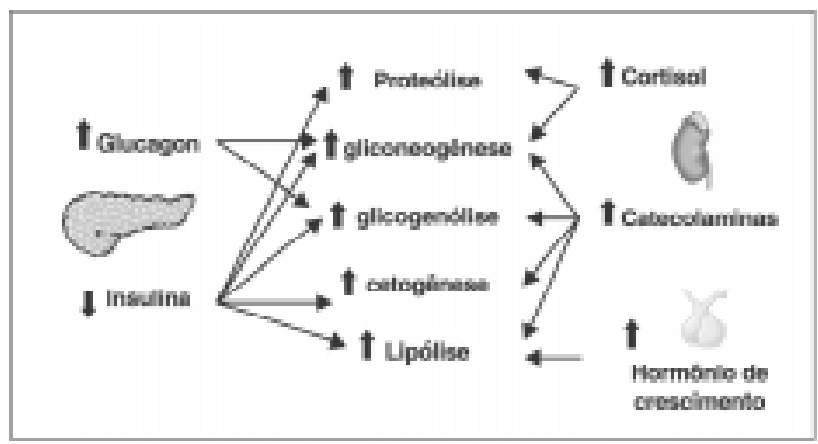

Figura 2 - Mecanismo de formação da acidose metabólica em CAD tecidos periféricos, ao mesmo tempo em que estimulam a produção de substratos para a gliconeogênese e cetogênese hepáticas.

\section{Quadro Clínico \\ História}

Os pacientes comumente apresentam uma história de poliúria e polidipsia devido à glicosúria. Pode ocorrer uma perda de peso, apesar de um aumento no consumo calórico, devido à perda excessiva de glicose pela urina e à incapacidade de se utilizar os substratos presentes na circulação.

Em pacientes sabidamente diabéticos, mas também em pacientes sem um diagnóstico prévio de diabetes mellitus, a história de um processo infeccioso é comum. Normalmente, em pacientes sem um controle adequado do diabetes, existe um equilíbrio metabólico delicado, e o stress de uma infecção quebra esse equilíbrio. Da mesma forma, pacientes sem o diagnóstico de diabetes mellitus conseguem se manter relativamente bem, apesar de uma progressiva perda da capacidade produtora de insulina. Com a infecção, a necessidade metabólica aumenta, e ocorre uma resistência transitória aos efeitos da insulina, fazendo com que, subitamente, o equilíbrio se perca e o quadro completo de CAD possa então se apresentar.

História de dor abdominal, vômitos e náusea também ocorre com freqüência. Esse quadro pode ser causado por um processo infeccioso ou pela perda do equilíbrio metabólico, já que a presença de corpos cetônicos plasmáticos em níveis elevados pode causar esses sintomas.

\section{Exame físico}

No exame físico as crianças em cetoacidose diabética apresentam-se abatidas, prostradas e desidratadas. A respiração característica (Kussmaul) pode estar presente em casos de acidose grave. Choque cardiovascular pode ocorrer em casos de desidratação extrema.

O exame abdominal deve ser feito com cuidado já que a apresentação pode ser muito semelhante à da apendicite aguda.

Um exame neurológico completo é necessário para se acompanhar a evolução do quadro e se diagnosticar complicações (edema cerebral) que podem vir a ocorrer com a terapia.

\section{Eletrólitos}

Devido à acidose metabólica e à poliúria, o organismo perde diversos eletrólitos $\left(\mathrm{Na}, \mathrm{K}, \mathrm{Cl}, \mathrm{PO}_{4}\right.$ e $\left.\mathrm{Ca}\right)$.

Sódio: a quantidade de sódio no corpo está diminuída e quando se mede o sódio plasmático, este não reflete precisamente o grau de deficiência. Esse erro ocorre devido à força osmótica da glicose. Para saber o valor real do sódio (sódio corrigido), usa-se a fórmula seguinte: para cada $100 \mathrm{mg} / \mathrm{dl}$ de glicose acima de 100 o sódio medido deve ser 


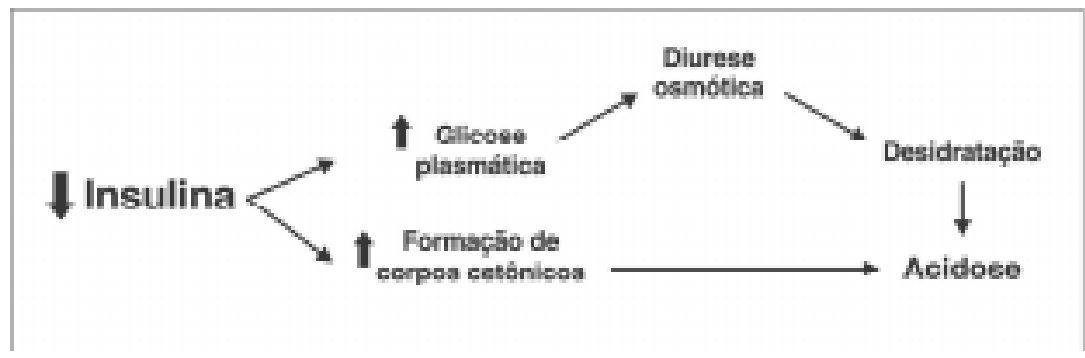

Figura 3 - Efeito hormonal no metabolismo energético

aumentado de 1,6 mEq/L. A fórmula final fica então: sódio corrigido $=[\mathrm{Na}]+1,6\{([$ glicose $]-100) / 100\}$. Por exemplo: Uma criança chega ao hospital com uma glicose de $900 \mathrm{mg} /$ dl e um sódio de $133 \mathrm{mEq} / \mathrm{L}$. À primeira vista, esta criança teria um sódio plasmático próximo dos limites inferiores na normalidade. Quando se corrige o sódio, observa-se que na verdade a criança está com um sódio plasmático acima dos valores superiores da normalidade. Usando a fórmula $o$ valor do sódio corrigido é de: $133+1,6\{(900-100) / 100\}=$ $145,8 \mathrm{mEq} / \mathrm{L}$.

Potássio: o corpo perde potássio devido à acidose e à poliúria. As células, para compensar a acidose extracelular, trocam íons $\mathrm{H}^{+}$por íons $\mathrm{K}^{+}$, perdendo grande quantidade de potássio. Os níveis de potássio sérico aumentam, e o paciente perde este potássio pela urina. Com isso, os níveis de potássio medidos no plasma, no momento da apresentação do paciente em CAD, podem ser baixos, normais, ou elevados, mas, com a correção da acidose metabólica, as células vão começar a liberar os íons $\mathrm{H}^{+}$e recuperar os íons $\mathrm{K}^{+}$, e o potássio plasmático vai diminuir. Os níveis plasmáticos diminuem aproximadamente $0,6 \mathrm{mmol}$ para cada 0,1 unidade de aumento no $\mathrm{pH}$.

\section{Complicações}

\section{Edema cerebral}

Edema cerebral sub-clínico pode estar presente antes de se iniciar o tratamento da $\mathrm{CAD}$, mas é uma complicação que normalmente só ocorre após a instituição da terapia com insulina. É uma complicação rara, ocorrendo em 1-3\% dos episódios de cetoacidose diabética, mas é responsável por mais de $30 \%$ das mortes ${ }^{5,7}$. É mais comum ocorrer em crianças com menos de 5 anos e em pacientes sem um diagnóstico prévio de diabetes (provavelmente devido à longa duração do distúrbio metabólico) $)^{5,12}$.

A etiologia do edema cerebral ainda não é bem conhecida. Níveis elevados de vasopressina e a presença de substâncias osmoticamente ativas no cérebro (produzidas em resposta ao aumento da pressão osmótica do sangue) são alguns dos fatores relacionados ao edema cerebral ${ }^{5,13}$. A maioria dos estudos mostra uma relação com alterações bruscas e acentuadas na pressão osmótica. Estudos retrospectivos mostraram que a administração excessiva de fluidos (acima de 4 litros $/ \mathrm{m}^{2} /$ dia), a diminuição rápida e acentuada da glicemia e a diminuição do sódio corrigido são fatores associados ao desenvolvimento de edema cerebral $^{15}$.

$\mathrm{O}$ uso de bicarbonato para corrigir mais rapidamente a acidose metabólica também está associado ao edema cerebral, provavelmente pela chamada acidose paradoxal, que representa uma piora transitória no $\mathrm{pH}$ cerebral após a administração de bicarbonato em pacientes com $\mathrm{CAD}^{13}$. O bicarbonato, após a administração venosa, reage com $\mathrm{H}^{+}$ presente na circulação, formando $\mathrm{H}_{2} \mathrm{O}$ e $\mathrm{CO}_{2}$ (que é então eliminado pela respiração), melhorando o $\mathrm{pH}$ do sangue periférico. Paradoxalmente, como o bicarbonato não atravessa facilmente a barreira hemato-encefálica e o $\mathrm{CO}_{2}$ atravessa, ocorre uma piora no $\mathrm{pH}$ a nível do sistema

Tabela 1 - Complicações mais comuns em crianças com CAD, com as respectivas causas e tratamento

\begin{tabular}{ccc}
\hline & Causa & Tratamento \\
\hline Choque cardiovascular & Desidratação & Soro fisiológico \\
Acidose severa & Desidratação e corpos cetônicos & Soro fisiológico e insulina \\
Hipopotassemia & Troca iônica entre os meios & Acrescentar potássio ao \\
& intra e extracelulares e poliúria & fluido de hidratação \\
Edema cerebral & Pressão osmótica? & Manitol, 1gm/kg rapidamente
\end{tabular}


Tabela 2 - Indicadores de risco de complicações durante o tratamento da cetoacidose diabética

Alterações do estado mental

$\mathrm{pH}$ arterial $<7,1$

Glicose plasmática $>1000 \mathrm{mg} / \mathrm{dl}$

$\mathrm{Na}>155 \mathrm{mEq} / \mathrm{L}$

$\mathrm{K}<3,5 \mathrm{mEq} / \mathrm{L}$

Idade $<5$ anos e especialmente $<1$ anos

Quando o sódio corrigido não aumenta com o tratamento

nervoso central. Essa piora transitória é, discutivelmente, a causa para uma maior incidência de edema cerebral nessa população. O problema dos estudos feitos mostrando uma relação entre o uso de bicarbonato e edema cerebral é que os pacientes que receberam bicarbonato eram também os pacientes com um quadro clínico mais severo, ficando difícil determinar se o uso de bicarbonato foi a causa do edema cerebral ou simplesmente foi usado em pacientes que iriam desenvolver edema cerebral de qualquer maneira.

O sinal clássico de edema cerebral é o desenvolvimento de hipertensão arterial associada à bradicardia. Outros sinais e sintomas são: alteração mental, aparecimento súbito de dor de cabeça severa, incontinência, vômitos, desorientação, oftalmoplegia, mudanças na aparência da pupila (assimetria, reação lenta ao estímulo luminoso, ou fixação), papiledema, convulsão e outras variações nos sinais vitais 7,17 .

O tratamento do edema cerebral consiste em aumentar rapidamente a pressão osmótica do plasma. Manitol $(0,25$ - $0,5 \mathrm{~g} / \mathrm{kg}$ ) deve ser usado em todos os pacientes com suspeita de edema cerebral. A causa dessa complicação ainda não foi esclarecida, mas a maioria dos estudos mostra que o melhor indicador de uma boa recuperação é a rapidez da equipe médica em iniciar a terapia com manitol. $\mathrm{O}$ uso de métodos de imagem para confirmar o diagnóstico somente aumenta o tempo entre o início dos sintomas e a terapia com manitol, aumentando os riscos de um resultado final não favorável. O serviço de neurocirurgia deve ser contactado rapidamente. O uso de hiperventilação é controverso nesses $\operatorname{casos}^{7,14,17}$.

\section{Terapia de CAD}

\section{Objetivos da terapia:}

corrigir a perda hídrica;

corrigir o déficit de insulina;

prevenir complicações.

\section{Exames laboratoriais no início da avaliação}

Glicose plasmática, eletrólitos, cálcio e fósforo devem ser dosados rotineiramente. A osmolalidade plasmática pode ser calculada pela fórmula: $(2[$ Sódio] + [uréa] $/ 2,8+$ [glicose]/18) e acompanhada em casos graves. Dependendo do quadro clínico, o $\mathrm{pH}$ venoso pode ser obtido inicialmente e, se acima de 7,1, dá a certeza de que o $\mathrm{pH}$ arterial está acima disso. Na presença de um quadro clínico mais grave ou de um $\mathrm{pH}$ venoso abaixo de 7,1, a obtenção de $\mathrm{pH}$ arterial se faz necessária. É importante lembrar que durante a primeira hora de terapia, existe uma piora no $\mathrm{pH}$, principalmente venoso, devido a uma "lavagem de ácido láctico" dos tecidos periféricos, que ocorre com a correção da desidratação.

O leucograma pode ajudar na investigação de uma causa infecciosa como origem da descompensação metabólica. Devido ao stress, uma leucocitose é comum durante a cetoacidose diabética, mas um desvio para a esquerda é mais comum na presença de uma infecção concomitante. $\mathrm{O}$ exame de urina ajuda no diagnóstico e deve ser feito regularmente como forma de acompanhamento da evolução do quadro, já que a concentração de corpos cetônicos na urina deve diminuir com uma terapia adequada com insulina.

\section{Tratamento \\ Líquidos}

No inicío, deve ser feita uma infusão venosa rápida (o mais rápido possível) de soro fisiológico (10-20 ml $/ \mathrm{kg})$. No caso do paciente se manter em estado de choque, mais soro fisiológico deve ser administrado na forma de infusão rápida. Maiores quantidades de fluidos não devem ser infundidas rapidamente devido ao risco de edema cerebral $^{5,10}$.

Após a estabilização do quadro clínico, líquidos de manutenção podem ser iniciados. Deve-se calcular o grau de desidratação da criança e o provável déficit hídrico. De maneira geral, calcula-se uma desidratação de $10 \%$ do peso corporal $^{21,23}$. O uso de parâmetros clínicos para estabelecer o grau de desidratação pode levar a erro, já que na CAD a desidratação é do tipo hiperosmolar. Desconta-se o volume de soro fisiológico administrado durante a fase de estabilização e divide-se o restante para ser administrado durante as próximas 48 horas. Acrescenta-se a este volume a taxa de manutenção. Este é o volume total de líquidos a ser administrado a cada hora. Inicia-se o "sistema de duas soluções" para reposição hídrica e controle da glicemia.

\section{O sistema de duas soluções}

Este sistema foi desenvolvido durante o uso (em pesquisa) de "clamps" hiperglicêmicos ou hiperinsulínicos. O principal objetivo é proporcionar à equipe médica uma maneira mais rápida e prática de fazer o controle glicêmi$\mathrm{co}^{4}$.

No método convencional de tratamento de CAD, necessita-se de diferentes soluções, com base na glicemia, já que a concentração de glicose a ser administrada varia. Neste 
sistema, prepara-se duas soluções diferentes, uma com glicose a 10 ou $12,5 \%$ e a outra sem glicose. A concentração salina de cada solução varia de acordo com o caso clínico.

Na Figura 3, mostra-se o sistema de duas soluções. Sabendo-se o volume total a ser administrado por hora, muda-se a relação entre a solução com glicose e a solução sem glicose com base na glicemia de cada momento. Em nosso serviço, preferimos usar concentrações diferentes de $\mathrm{NaCl}$ com base na idade do paciente e no sódio calculado. Outros serviços usam somente soro fisiológico, para garantir uma elevação no sódio corrigido ${ }^{23}$.

Com o uso deste sistema, alterações na concentração da glicose administrada podem ser feitas instantaneamente, ao invés de esperar pela preparação de um nova solução com a concentração de glicose calculada. Ao mesmo tempo, alterando-se a relação entre as duas soluções, pode-se conseguir qualquer concentração de glicose que se deseje.

As concentrações de $\mathrm{NaCl}, \mathrm{KCl}$, e $\mathrm{K}_{2} \mathrm{PO}_{4}$ de cada soro, assim como a concentração de glicose infundida a cada momento podem ser adaptadas para cada serviço, com base em experiências pessoais.

\section{O controle da glicemia com o sistema de duas soluções}

A glicemia deve ser monitorada a cada hora através da análise no sangue capilar. A queda da glicemia não deve ser maior do que $50 \mathrm{mg} / \mathrm{dl} /$ hora após as 2 primeiras horas de tratamento, e a queda total, nas 6 primeiras horas, não deve ser superior a $600 \mathrm{mg} / \mathrm{dl}$. A adição de glicose ao fluido intravenoso é baseada na glicemia inicial e na glicemia de cada hora.

Quando a glicemia inicial é acima de $800 \mathrm{mg} / \mathrm{dl}$, recomenda-se começar glicose intravenosa quando a glicemia capilar estiver abaixo de $500 \mathrm{mg} / \mathrm{dl}$. Já quando a glicemia de apresentação for abaixo de $800 \mathrm{mg} / \mathrm{dl}$, adiciona-se glicose ao líquido intravenoso quando a glicemia capilar estiver abaixo de $350 \mathrm{mg} / \mathrm{dl}$. A Tabela 3 relaciona a concentração de glicose no líquido intravenoso e a glicemia capilar inicial e a de cada hora. Esses valores são baseados na nossa experiência, mas diferentes serviços podem usar diferentes valores.
Por exemplo: uma criança que pese $30 \mathrm{~kg}$ e está $10 \%$ desidratada recebeu $20 \mathrm{ml} / \mathrm{kg}$ de soro fisiológico como infusão rápida. $\mathrm{O}$ volume de manutenção (baseado no peso) é de $70 \mathrm{ml} /$ hora. A perda hídrica era de 3,0 litros, e ela já recebeu $600 \mathrm{ml}$. Falta repor 2,4 litros. Calculando-se esta reposição em 48 horas, chega-se a um volume de $50 \mathrm{ml} /$ hora. Somando-se o volume de reposição ao volume de manutenção, tem-se que o volume total é de $120 \mathrm{ml} /$ hora. Se a glicemia de apresentação era de $935 \mathrm{mg} / \mathrm{dl}$ e a glicemia atual é de $427 \mathrm{mg} / \mathrm{dl}$, deve-se dar $90 \mathrm{ml} /$ hora $(75 \%$ do volume total) da solução sem glicose e $30 \mathrm{ml} /$ hora (25\% do volume total) da solução com glicose. Se a glicemia for de $370 \mathrm{mg} / \mathrm{dl}$, altera-se a relação entre as duas soluções para aumentar a oferta de glicose, mantendo-se o volume total. Com isso, deve-se dar $60 \mathrm{ml} /$ hora de cada uma das soluções.

\section{Insulina}

Se a criança apresenta um quadro de CAD leve, insulina de ação rápida (lispro) por via subcutânea pode ser usada a cada hora ou pode-se usar insulina regular por via intramuscular. Em quadros mais graves, assim que o paciente é identificado como em CAD, deve-se preparar insulina

Tabela 3 - Demonstração da quantidade de glicose infundida, baseada na glicemia de apresentação (acima ou abaixo de $800 \mathrm{mg} / \mathrm{dl}$ ) e na glicemia horária

\begin{tabular}{|c|c|c|c|}
\hline \multicolumn{2}{|c|}{ Glicose inicial } & \multicolumn{2}{|c|}{$\begin{array}{c}\text { \% líquido total } \\
\text { (manutenção + déficit) }\end{array}$} \\
\hline$>800 \mathrm{mg} / \mathrm{dl}$ & $<800 \mathrm{mg} / \mathrm{dl}$ & Sol. sem & Sol. com \\
\hline \multicolumn{4}{|c|}{ Glicose atual } \\
\hline$>501 \mathrm{mg} / \mathrm{dl}$ & $>350 \mathrm{mg} / \mathrm{dl}$ & $100 \%$ & zero \\
\hline $401-500 \mathrm{mg} / \mathrm{dl}$ & $301-350 \mathrm{mg} / \mathrm{dl}$ & $75 \%$ & $25 \%$ \\
\hline $301-400 \mathrm{mg} / \mathrm{dl}$ & $251-300 \mathrm{mg} / \mathrm{dl}$ & $50 \%$ & $50 \%$ \\
\hline $201-300 \mathrm{mg} / \mathrm{dl}$ & $201-250 \mathrm{mg} / \mathrm{dl}$ & $25 \%$ & $75 \%$ \\
\hline$<200 \mathrm{mg} / \mathrm{dl}$ & $<200 \mathrm{mg} / \mathrm{dl}$ & zero & $100 \%$ \\
\hline
\end{tabular}

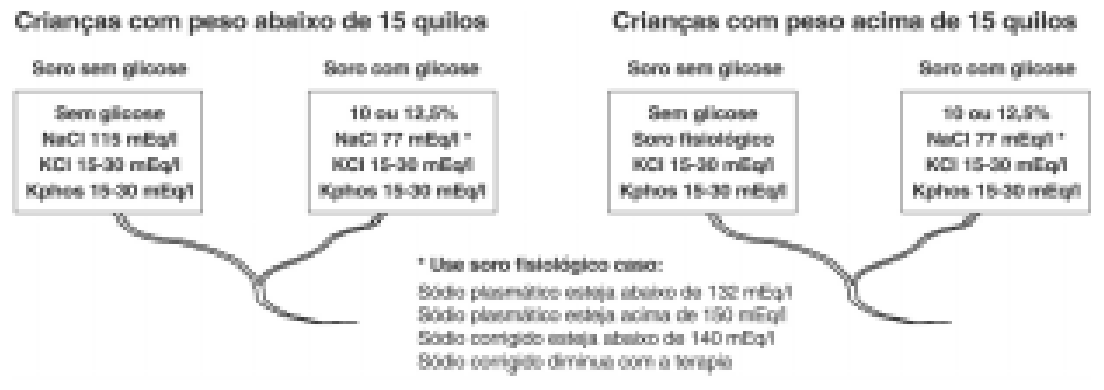

Figura 4 - Demonstração gráfica do "sistema de duas soluções" 
regular para infusão intravenosa numa velocidade de 0,05 - 0,1 u/kg/hora ${ }^{5,10,21-26}$. Esta insulina deve ser iniciada o mais rápido possível, exceto em quadros de hiperosmolaridade com uma acidose metabólica mínima ( $\mathrm{pH}$ acima de $7,25)$, que pode acontecer principalmente em pacientes com diabetes mellitus tipo 2. Nestes casos, o risco de complicações decorrentes de uma queda aguda na glicemia causada pela insulina é maior do que a necessidade de se eliminar o processo cetótico (se presente).

No caso de pacientes em que a hiperglicemia já foi corrigida mas a acidose metabólica continua presente, considerando que a insulinoterapia é essencial, aumenta-se a concentração de glicose do líquido administrado para $12,5 \%$ ou mais se um acesso venoso central estiver presente.

\section{Hypoglicemia}

No caso de uma hipoglicemia (glicose plasmática abaixo de $60 \mathrm{mg} \%$ ) ocorrer, e o paciente não apresentar sintomas, aumenta-se a infusão de glicose em $25 \%$.

Se o paciente estiver inconsciente ou tendo uma convulsão, deve-se administrar $1-2 \mathrm{ml} / \mathrm{kg}$ de glicose $25 \%$ por via venosa. Essa medida rápida deve ser seguida por um aumento na infusão de glicose em $25 \%$. Se a acidose metabólica estiver corrigida, pode-se diminuir a infusão de insulina em $25 \%$.

\section{Uso de bicarbonato de sódio}

$\mathrm{O}$ uso de bicarbonato é controverso devido a diversos fatores $^{5,10,21-26}$. A correção do $\mathrm{pH}$ sangüíneo com bicarbonato traria como vantagens a melhora da contractilidade cardíaca e a diminuição da incidência de arritmias ventriculares devido à acidose. Por outro lado, como já foi mencionado anteriormente, o uso de bicarbonato pode estar associado a uma maior incidência de edema cerebral e pode piorar a hipopotassemia, diminuir os níveis de cálcio ionizado e causar um aumento na afinidade entre hemoglobina e oxigênio, causando hipóxia tecidual. Recomenda-se somente quando o $\mathrm{pH}$ arterial estiver abaixo de 7,0 - 7,1 ou quando a compensação respiratória (alcalose respiratória) for inadequada 7 .

Bicarbonato é administrado lentamente na dose de 1-2 $\mathrm{mEq} / \mathrm{kg}$ por via venosa, levando-se aproximadamente 2-4 horas para a administração da dose total.

\section{Acompanhamento laboratorial}

A glicose plasmática deve ser medida a cada 1 hora. Eletrólitos devem ser acompanhados a cada 2-4 horas dependendo do estado do paciente na apresentação e dependendo da evolução clínica ${ }^{5,23-26}$. A não ser em casos graves ou raros, não há necessidade de repetir os níveis plasmáticos de cálcio e fósforo ou o leucograma.

\section{Conclusões}

A melhor terapia para a cetoacidose diabética é a prevenção. Praticamente todos os casos de cetoacidose diabética em crianças sabidamente diabéticas podem ser evitados com um acompanhamento regular.

A correção do distúrbio metabólico/ hídrico presente nos quadros de cetoacidose diabética deve ser feita de maneira conservadora e lenta, a não ser nos casos extremos.

O uso de insulina é essencial para a correção da cetoacidose metabólica, diferentemente de pacientes com diabetes mellitus tipo 2, em que com o uso de líquidos ocorrerá uma melhora grande.

Apesar de não existir, no momento, uma prova concreta de que a queda do sódio corrigido cause edema cerebral, a demonstração de uma associação faz com que este seja acompanhado cautelosamente.

Como foi dito anteriormente, o objetivo desta revisão não é mostrar um novo regime terapêutico para o tratamento da CAD, mas sim mostrar uma maneira de tornar o controle mais prático de modo a poder ser adaptado para cada serviço.

\section{Referências bibliográficas}

1. Sperling MA. Aspects of the etiology, prediction, and prevention of insulin-dependent diabetes mellitus in childhood. Pediatric Clinics of North America 1997; 44:269-84.

2. Edge JA. Dunger DB. Variations in the management of diabetic ketoacidosis in children. Diabetic Medicine 1994; 11:984-6.

3. Glaser NS, Kuppermann N, Yee CK, Schwartz DL, Styne DM. Variation in the management of pediatric diabetic ketoacidosis by speciality training. Archives of Pediatrics \& Adolescent Medicine 1997; 151:1125-32.

4. Grimberg A, Cerri RW, Satin-Smith M, Cohen P. The "two bag system" for variable intravenous dextrose and fluid administration: benefits in diabetic ketoacidosis management. Journal of Pediatrics 1999; 134:376-8.

5. Lebovitz HE. Diabetic ketoacidosis. Lancet 1995; 345 (8952): 767-72.

6. Wetterhall SF, Olson DR, DeStafano F, et al. Trends in diabetes and diabetic complications, 1980-1987. Diabetes Care 1992; 15:960-6.

7. Rosenbloom AL, Hanas R. Diabetic ketoacidosis (DKA): treatment guidelines. Clinical Pediatrics 1996; 35:261-6.

8. Smith CP, Firth D, Bennett S, Howard C, Chisholm P. Ketoacidosis occurring in newly diagnosed and established diabetic children. Acta Paediatrica 1998; 87:537-41.

9. Morris AD, Boyle DI, McMahon AD, Greene SA, MacDonald TM, Newton RW. Adherence to insulin treatment, glycaemic control, and ketoacidosis in insulin-dependent diabetes mellitus. The DARTS/MEMO Collaboration. Diabetes Audit and Research in Tayside Scotland. Lancet 1997; 350(9090):1505-10.

10. Hoffman WH, Steinhart CM, el Gammal T, Steele S, Cuadrado AR, Morse PK. Cranial CT in children and adolescents with diabetic ketoacidosis. American Journal of Neuroradiology 1988; 9:733-9. 
11. Krane EJ, Rockoff MA, Wallman JK, Wolfsdorf JI. Subclinical brain swelling in children during treatment of diabetic ketoacidosis. New England Journal of Medicine 1985; 312:1147-51.

12. Rosenbloom AL. Intracerebral crises during treatment of diabetic ketoacidosis. Diabetes Care 1990; 13:22-33.

13. Krane EJ. Diabetic ketoacidosis and cerebral edema. http:// PedsCCM.wustl.edu/FILE-CABINET/Metab/DKACEdema.html. 1996.

14. Klekamp J, Churchwell KB. Diabetic ketoacidosis in children: initial clinical assessment and treatment. Pediatric Annals 1996; 25:387-93.

15. Duck SC, Wyatt DT. Factors associated with brain herniation in the treatment of diabetic ketoacidosis. Journal of Pediatrics 1988; 113 (1 Pt1):10-4.

16. Finberg L. Why do patients with diabetic ketoacidosis have cerebral swelling, and why does treatment sometimes make it worse? Archives of Pediatrics \& Adolescent Medicine 1996; 150:785-6.

17. Harris GD, Fiordalisi I, Harris WL, Mosovich LL, Finberg L. Minimizing the risk of brain herniation during treatment of diabetic ketoacidemia: a retrospective and prospective study. Journal of Pediatrics 1990; 117(1 Pt1):22-31.

18. Hale PM, Rezvani I, Braunstein AW, Lipman TH, Martinez N, Garibaldi L. Factors predicting cerebral edema in young children with diabetic ketoacidosis and new onset type I diabetes. Acta Paediatrica 1997; 86:626-31.

19. Silver SM, Clark EC, Schroeder BM, Sterns RH. Pathogenesis of cerebral edema after treatment of diabetic ketoacidosis. Kidney International 1997; 51:1237-44.

20. Mahoney CP, Vlcek BW, DelAguila M. Risk factors for developing brain herniation during diabetic ketoacidosis. Pediatric Neurology 1999; 21:721-7.

21. Kecskes SA. Diabetic ketoacidosis. Pediatric Clinics of North America 1993; 40:355-63.
22. Harris GD, Fiordalisi I. Physiologic management of diabetic ketoacidemia. A 5-year prospective pediatric experience in 231 episodes. Archives of Pediatrics \& Adolescent Medicine 1994; 148:1046-52.

23. Rosenbloom AL, Schatz DA. Diabetic ketoacidosis in childhood. Pediatric Annals 1994; 23:284-8.

24. Edge JA. Management of diabetic ketoacidosis in childhood. British Journal of Hospital Medicine 1996; 55:508-12.

25. Silink M. Practical management of diabetic ketoacidosis in childhood and adolescence. Acta Paediatrica 1998; 425:63-6.

26. Kitabchi AE, Wall BM. Management of diabetic ketoacidosis. American Family Physician 1999; 60:455-64.

27. Viallon A, Zeni F, Lafond P, Venet C, Tardy B, Page Y, Bertrand JC. Does bicarbonate therapy improve the management of severe diabetic ketoacidosis? Critical Care Medicine 1999; 27:2690-3.

28. Hale D. In: Fleisher GF, Ludwig S, eds. Textbook of Pediatric Emergency Medicine, $4^{\text {th }}$ ed. Philadelphia: Lippincott Williams \& Wilkins; 2000. p.1096.

Endereço para correspondência:

Dr. Paulo Ferrez Collett-Solberg

Division of Pediatric Endocrinology

Duke University Medical Center

308 Bell Building, Box 3080

Durham, NC 27710 - USA

E-mail: colle002@mc.duke.edu 